\author{
Section 11. Organic Molecules
}

\title{
EXCITON DYNAMICS IN THIN WIRES *
}

\author{
Raoul KOPELMAN, Li LI, Stephan J. PARUS and Jagdish PRASAD \\ Department of Chemistry, University of Michigan, Ann Arbor, MI 48109, USA
}

\begin{abstract}
When are molecular wires thin enough to show one-dimensional exciton kinetics? What are the characteristics of one-dimensional kinetics? What applications are there? Cylindrical naphthalene wires ( 5-5000 nanometer radius) show a definite one- to three-dimensional transition (about $25 \mathrm{~nm}$ for triplet excitons at $4 \mathrm{~K} ; 40 \mathrm{~nm}$ at $77 \mathrm{~K}$ ). Nuclear channel pore membranes (polycarbonate) serve as templates and calibrators. Monte Carlo simulations on finite-width wires are consistent with the experiments. $\mathrm{Vycor}$ glass pores are effectively one-dimensional. A new experimental criterion is based on excitation pulse width. It gives both topological and stochastic information (i.e. dimensionality and hopping rate). Its applicability to ultrathin wires and porous glass is demonstrated via simulations and experiments. The triplet exciton migration (multiple hopping) length is $50-100$ molecules for all samples.
\end{abstract}

\section{Introduction}

Solid-state and stochastic problems in one dimension have been of long-standing theoretical interest [1-5]. Currently, electronics in thin wires is of much theoretical and practical interest [6-9]. The theoretical enigmas (localization, mesoscopic phenomena, boundary effects) are compounded by experimental difficulties: minute currents, heat dissipation, shorts, non-uniformity and suspect testing procedures. Many of these difficulties are not present with Frenkel excitons. There are no Coulomb repulsions and for triplet excitons the interactions are extremely short-ranged and the surface effects are minimal $[4,10]$. Moreover, triplet excitons are already localized in the bulk [11] and thus there is no localization-delocalization transition or cross-over. Experimentally one can rely on optical measurements which are as simple for thin wires as for the bulk. Furthermore, sample uniformity or continuity is not a crucial factor. One can thus concentrate on the mesoscopic properties of interest, stemming from the confinement of the excitons inside a thin "wire".

Recently, porous materials and "fractal" networks have also been of much interest [12-15]. The difference between a fractal network and a quasi-one-dimensional network is often not very clear [12-16]. Energy transfer [13,14] and exciton kinetics [15] have been used for the characterization of such networks (e.g. pore networks of porous media). Understanding the characteristics of truly one-dimensional networks and the effects of sample diameter is thus of practical interest. Furthermore, molecular of polymeric chains, fibers, filaments and networks exist in most synthetic, natural and biological organic systems, from organic conductors to neuron transmitters. Molecular exciton kinetics in thin filaments are of relevance to all these systems.

We note that our system differs significantly from pre-

* Supported by NSF Grant No. DMR-83-03919. viously studied quasi-one-dimensional systems [17-20]. The latter are essentially two or three-dimensional systems with highly anisotropic exciton-exchange interactions. Thus, for a short time the exciton is confined in one-dimension. However, there is always a finite probability of moving along other directions (interchain hopping), resulting in a two- or three-dimensional behavior over longer times (this usually confines the measurements to ultra-short times). Moreover, the phonons and exciton-phonon interactions in these systems are seldom one-dimensional. In contrast, our systems are truly onedimensional over long times and there is no escape or tunneling out of the thin, one-dimensional systems. (Our ultrathin wires are obviously three-dimensional on extremely short time scales.)

Exciton transport is usually monitored via the kinetics of trapping or annihilation $[11,15,18]$. The kinetic process may be unary, pseudo-unary or binary (monomolecular, pseudo-monomolecular or bimolecular in chemical language), e.g. trapping, heterofusion or homofusion, respectively $[4,15,21]$. In all these cases the "rate-constant" (instantaneous reactive collision probability per unit density) is given by [21]

$k \sim \mathrm{d} S / \mathrm{d} t$,

where $S$ is the mean number of distinct lattice sites visited by a single exciton (in the absence of reactive processes). We note that eq. (1) is valid for all topologies, in contrast to the expression $k \sim D(D=$ diffusion constant) which is valid only for 3-dimensional (homogeneous) lattices. To a very good approximation one has [21]:

$S \sim t^{\prime} \quad 1 / 2 \leqslant f \leqslant 1 ;$

$k \sim t^{-h} \quad 1 / 2 \leqslant h=1-f \leqslant 1$.

At long times $h=0$ for three-dimensional lattices and $h=1 / 2$ for one-dimensional lattices. We note that for fractal lattices $h=1-d_{\mathrm{s}} / 2$ where $d_{\mathrm{s}}$ is the spectral dimension $[21-23]$. The pragmatic questions we pose are: How 
thin has a wire to be to give one-dimensional behavior $(h=1 / 2)$ ? What is the nature of the crossover from threedimensional to one-dimensional behavior? What does this crossover depend on? What can we learn from it? What is the use of thin exciton wires?

We note here that in perfect crystalline samples the excitons move freely (at random, due to phonons), resulting in binary (bimolecular) exciton-exciton annihilation [21.24]:

$A+A \rightarrow h w^{\prime}$.

where $h \nu$ designates fluorescence ("delayed"). Thus the annihilation rate $R$ and the fluorescence rate $F$ are given by [24]

$F \sim R=k \rho$

where $\rho_{i}$ is the free exciton density. However, in most real samples [15,25], a fraction of the free excitons (.1) are quickly trapped, giving a roughly constant density $p_{1}$, of trapped excitons $\left(A^{\prime}\right)$, resulting in a pseudo-unary (pseudo-monomolecular) annihilation reaction and rate:

$A+A^{\prime} \rightarrow h h^{\prime}$.

$F \sim R=k^{\prime} \rho_{1} \quad k^{\prime}=k \rho_{1}$.

In addition. the triplet excitons undergo natural decay (lifetime $\tau$ ):

$A \rightarrow h l: \quad P=\tau \quad p_{1}$.

The overall results are thus:

$k \sim F / P^{n} \quad n=1,2$.

where $n=2$ is for perfect (trapless) samples and $n=1$ is for real samples (with traps). We note that for three-dimensional samples $k$ (and $\log k$ ) is expected to be constant in time while for one-dimensional samples $k \sim t^{-1: 2}$ and $\log k \sim \log t$ (with a slope of $-1 / 2$ ).

Exciton and electron-hole recombination kinetics are believed $[4,25-27]$ to depend only on the initial density. for a given sample and given thermodynamic parameters (temperature). The same goes for surface and solid state reactions [27,28], including defect aggregation [29]. Whether the cnsemble has been prepared under steadystate conditions (e.g. cw excitation) or as a random ensemble (e.g. pulse excitation) was believed not to matter. as long as the global density is the same at initiation time $(t=0)$. We show that the above assumption works quite well for homogeneous samples, but not for heterogeneous or low-dimensional samples. Specifically, for some fractal-like, quasi-linear or dispersed samples the local densilies and pair-correlation functions determine the decay functions, especially at early times. Such experiments can thus serve as probes for the dimensionality as well as the geometric and/or energetic heterogeneity of the sample. They can also serve as indicators of diffusive vs. dispersive motion, of the degree of motional coherence and of the degree of stirring and self-stirring of the ensemble. This method may also provide an absolute calibration for the hopping time.

\section{Experimental}

Experiments were carried out on naphthalene impregnated porous glass (Vycor), porous polymeric membranes, naphthalene powder [15.21], and perfectly crystalline isotopic alloys of naphthalene [21,24]. The purification, preparation and other experimental procedures were the same as in previous studies $[15.21,24,30]$. The random population of triplet naphthalene excitons was produced by "pulse" excitation: msec duration pulses produced via mechanically shuttered xenon lamp excitation $[15.21,24.30]$. The steady state population was created via excitation by the same xenon lamp. but the lamp was "on" till well after a steady state phosphorescence signal was established (several seconds). and then shuttered "off". Neutral density filters insured equal phosphorescence signals at time "zero" (the closing time of the shutters in both excitation modes). Both phosphorescence and delayed fluorescence decays are quite different for the pulsed vs. steady-state excitation mode. especially at early times. Fig. I shows phosphorescence and fluorescence decays for both pulsed and steady-state excitations of naphthalene embedded porous glass. Similar results are obtained for naphtalene powders, and for low concentration naphthalene isotopic alloys ("below percolation" "). On the other hand, no such differences in decay curves can be seen for high concentration alloys ("above percolation") or nearly perfect naphthalene crystals [30].

Significant is the use of channel-pore ("nuclepore") [31] polycarbonate membranes. These 6 micron thick membranes come with well isolated, clindrical pores (fig. 1 of ref. [32]). While a given membrane has uniform pore diameters, membranes with different pore diameters are available and we used them in the range of $10 \mathrm{~nm}(100$ A) to one micron $(10000 \AA$ ). Some typical results are shown in fig. $2(T=4 \mathrm{~K})$. We note that only the pseudounary model $(n=1)$ resulted in linear slopes. The binary model $(n=2)$ cannot be fitted with straight lines and. moreover, results in non-constant $k$ curves even for the thickest wires $(1.2 \mu \mathrm{m})$. The totality of the $h$ values (negative slopes), for all wires (each at 4 and $77 \mathrm{~K}$ ), is given in fig. 3 .

\section{Monte Carlo simulations}

A system of random walkers is created on various lattices. In one case (I) the walker population is created via a random placement of particles on the lattice. This mimics the creation of naphthalene excitons via "pulse" (the weak optical absorption of photons is presumed to be random over the thin sample). When two walkers collide 


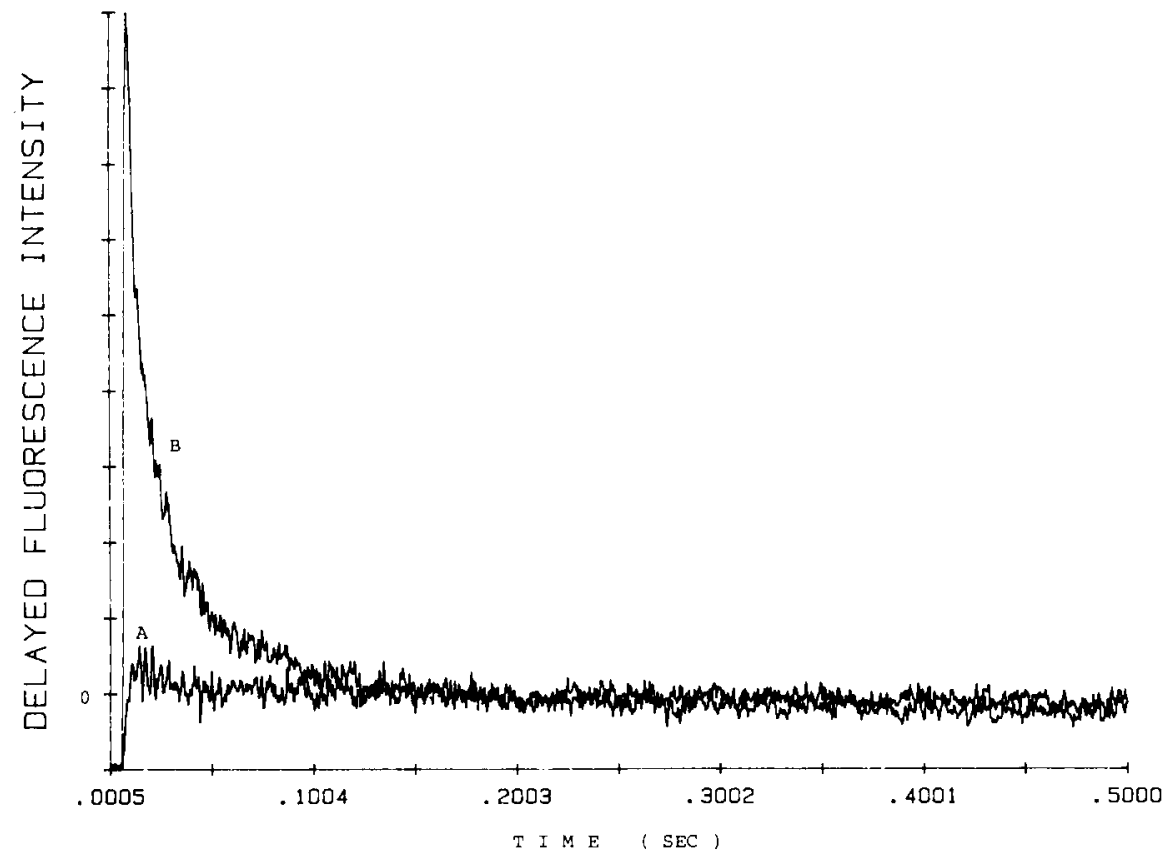

Fig. 1. Delayed fluorescence decay from naphthalene in Vycor glass at $6 \mathrm{~K}$ following excitation ( $310 \mathrm{~nm}$ ) durations of (A) $11 \mathrm{~s}$; (B) 20 ms. The excitation of (A) had a neutral density filter of 1.2 to equalize the initial phosphorescence intensity of (A) and (B).

they annihilate: $A+A \rightarrow 0$. This mimics the exciton reaction: triplet + triplet $\rightarrow h \nu$, where $h \nu$ is fluorescence ("delayed"). (The "natural decay" (phosphorescence) of the walkers has been incorporated in some simulations, but plays a minor role due to the relatively long triplet lifetime). For the second case (II), the steady state excita- tion is simulated via a steady rate of particle creation. The particles move at random and annihilate $(A+A \rightarrow 0)$. After a while a steady state density of walkers is established $[33,34]$. The creation of particles is stopped at a time defined as "zero". The random walk and annihilation continue and are monitored. Case $I$ (random creation) is

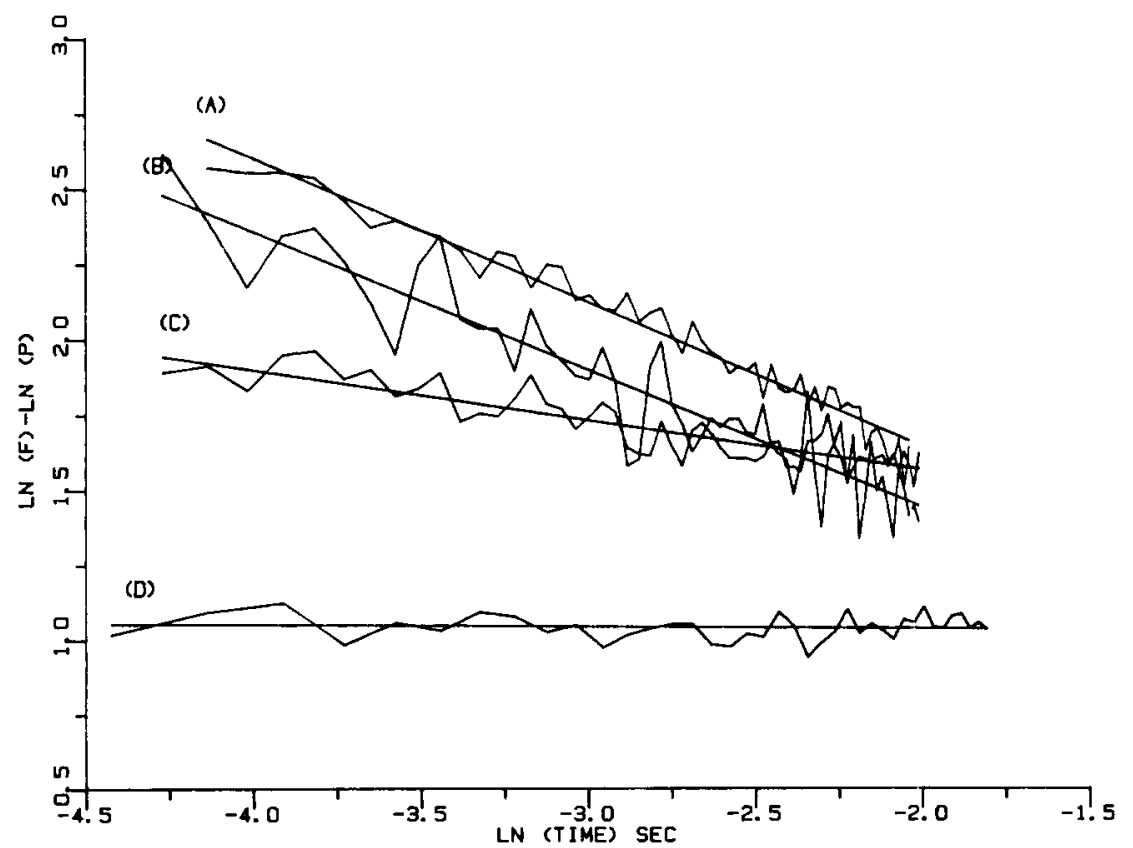

Fig. 2. Annihilation rate coefficient $R=F / P$ vs. time on a $\ln -\ln$ scale, for naphthalene filled channel-pore polycarbonate membranes at $T=4 \mathrm{~K}$. The pore diameters are (A) $150 \AA$, (B) $300 \AA$ (C) $500 \AA$ and (D) $800 \AA$. Note that the trapped (bound) exciton phosphorescence is excluded via an interference filter (centered at the free exciton peak). 


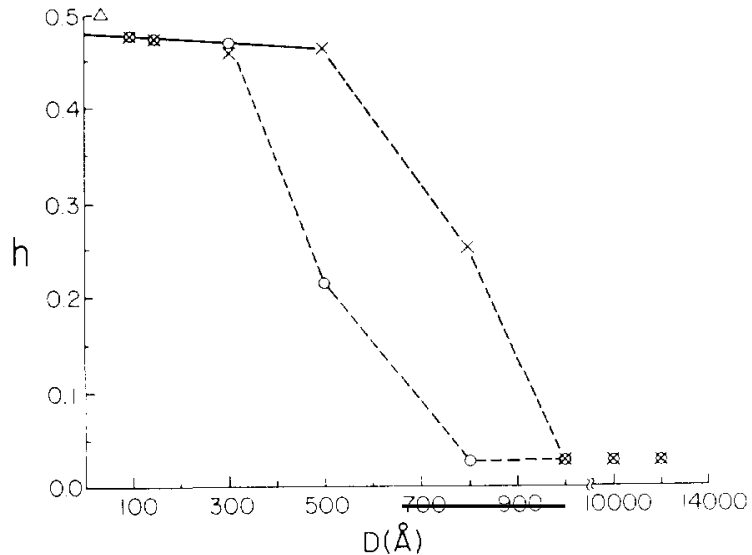

Fig. 3. Exponent his, wire diameter (in angstrom) a $4 \mathrm{~K}(\bigcirc)$ and $77 \mathrm{~K}(\mathrm{X})$. The 20 A point $(\triangle)$ is for porous vycor (ref. [15]). Note break in scale.

normalized by starting with the same glohal density (number of particles) as Case II (at time "zero"). We note that while the initial global densities (monitored cxperimentally via phosphorescence) are equal in both Case I and II (for both simulation and experiment), this does not guarantee an equal annihilation rate (delayed fluorescence) at time "zero" (or at any other time). The annihilation rate depends on the local densities.

\section{Results and discussion}

We have simulated diffusion-limited reactions on true one-dimensional systems and also on quasi-one-dimensional systems such as two- and three-dimensional "wires" (sec fig. 1 of ref. [35]). For instance. for the reaction $A+A \rightarrow 0$ we find an initial classical behavior, followed quickly by a one-dimensional behavior (for pulsed reactions). For long times we find $h \approx 0.5$. for both geminate and non-geminate generation of 4 . These are the same results as for a strictly one-dimensional chain [33]. We conclude that the typical one-dimensional behavior for annihilation reactions is preserved for one-dimensional wires with finite thickness, for both "flat" and "round" wires. This is true as long as the length of the wire is large compared to its cross-section and provided that the observation time is long compared with the time it takes a particle to reach the edge of the wire [35].

For the naphthalene wires in the cylindrical polycarbonate membranes (figs. 2,3 ) we observed that the thinnest wires yicld a value $h \approx 0.5$, while the thickest wires give $h \approx 0$. for both temperatures. Actually, extrapolating (1) zero diameter. $h \rightarrow 0.49 \pm 0.02$. On the other hand. for micron sized wires $h \rightarrow 0.02 \pm 0.02$. These two limiting values are in excellent agreement with the theoretically expected values of $h=1 / 2$ and $h=0$, respectively. The cross-over (between $h \approx 0$ and $h \approx 1 / 2$ ) occurs at diameters of about 500 to $800 \AA$ at 4 and $77 \mathrm{~K}$, respectively. The cross-overs are relatively sharp and their tempera- ture dependence is relatively mild. The higher value at higher temperatures is consistent with a somewhat faster hopping rate.

In our interpretation the cross-over radius is roughly consistent with the average (ruising range $\lambda$ (end-point to end-point distance) of the exciton, within its lifetime'. An indirect. rough estimate for the naphthalene cruising range in similarly prepared samples was given [15] as $\lambda=1000$ A. This is in excellent agreement with our present result $(\lambda=500 A)$. Obviously, for wires with radius $r \gg \lambda$ the excitons do nol "feel" the pore boundaries while for $r \ll \lambda$. the excitons are severely confined along two of the three directions. We note that the polycarbonate excitation energy values are so much higher than those of naphthalene that there is a vanishing probability for barrier crossing or tunneling $(\Delta E>100 \mathrm{kT}$ even at $77 \mathrm{~K}$ ).

Of particular interest is the resolution of the porous glass (Vycor) dilemma [15]. The nature of the pore network has been highly controversial [12-16]. It has been argued on one hand that it is a random (percolation-like) network with a fractal dimension on the order of two [13]. On the other hand it has been argued to be non-fractal but essentially onc-dimensional $[12,15.16]$. Using the exciton kinetics technique it was argued [15] that the effective spectral dimension is 1.05 . i.e. effectively onedimensional. Our present study uses the same approach for "calibrated" cylindrical pores which are obviously nonfractal and one-dimensional. We have included the older [15] Vycor glass measurements as a data point in fig. 3 . It essentially falls on the same curve as the new. polycarbonate data. The Vycor data are thus totally consistent with a one-dimensional pore topology. Further proof is given below.

The naphthalene-embedded porous Vycor data of fig. 1 are now replotted (fig. 4 in a ratio plot of the annihilation rates (pulse-generated over steady-state-generated delaled fluorescence). We note the high initial (short time) value of this ratio (about seven). It turns out that only with one-dimensional topologies can one achieve such high initial ratios. For comparison, fig. 4 gives the simulation results for a number of topologics. A cubic topology results in an effective ratio of unit!, the classically expected value. Three-dimensional topologies of percolation clusters (including the whole ensemble of percolation islands! ) and monodisperse cubic islands $(3 \times 3 \times 3)$ give ratios higher than unity but lower than five. The same is true for two-dimensional topologies (including fractals, e.g. Sierpinski carpets, critical percolation clusters and the monodisperse square islands). Only the one-dimensional topologies (continuous and disperse) give curves resembling the experimental data (fig. 4 ).

The above experimental data appear to require a wirelike topology. with at least partial breaks (an assembly of norms is a good picture). Independent knowledge of the exciton hopping time would narrow down the topology (giving an effective "worm-length"). On the other hand, the present information suffices to constrain the average 


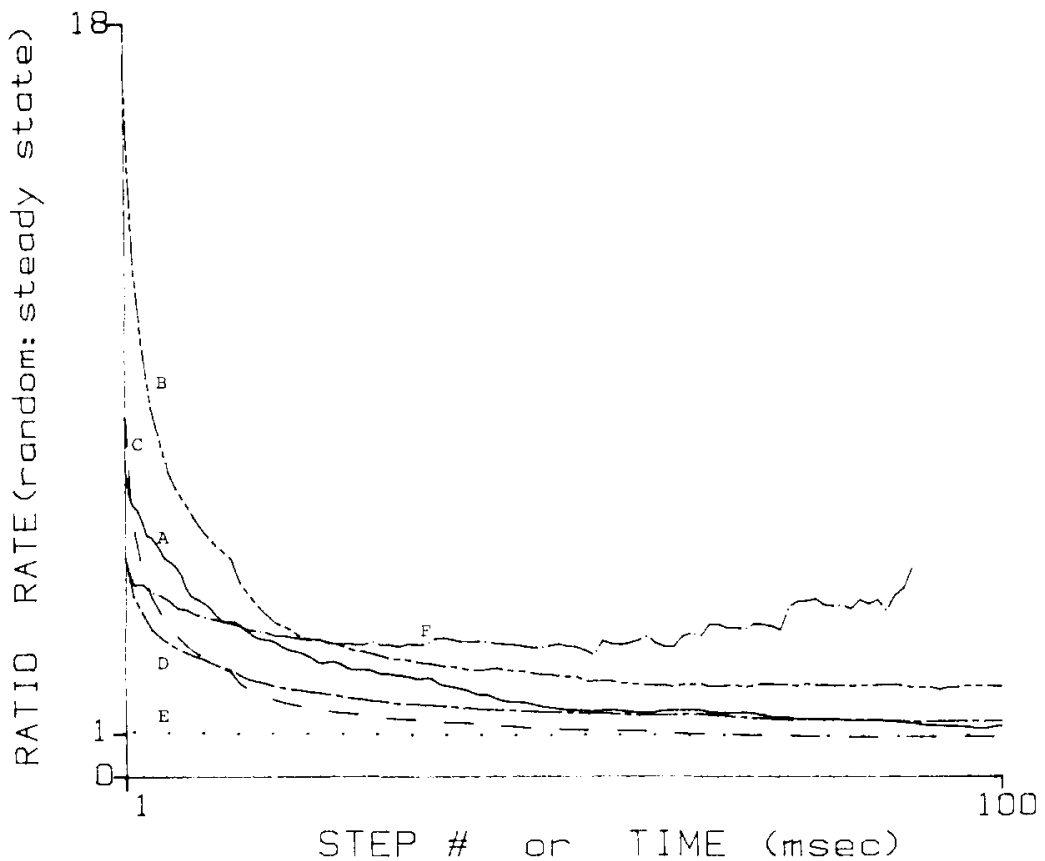

Fig. 4. Ratio of annihilation rates (pulsed over steady-state generation) vs. time: (A) Vycor glass (see fig. 1) with time in ms. (B) Onedimensional islands (20 sites each). (C) One-dimensional chain. (D) Threc-dimensional percolation clusters (cubic, $40 \%$ occupation. all clusters). (E) Three-dimensional lattice (cubic). (F) Three-dimensional islands $(3 \times 3 \times 3$ sites each). Note: 1 . Each simulated ensemble includes about 30000 sites. 2. Simulation time in number of steps.

individual hopping time to about $1 \mathrm{~ms}$ (certainly within an order of magnitude). This translates into an effective cruising length of about 50 molecules or about 250 to 400 $\AA$. This is in good agreement with the polycarbonateembedded wires (see above) and our previous estimate [15].

We have argued above, based on a different kind of kinetic parameter (the heterogeneity exponent for long time decays), that naphthalene channels in porous Vycor are quasi-one-dimensional. Our present kinetic data (based on local densities at early decay times) appear to be consistent with the above picture and with other work [12]. More importantly, the high value of the heterogeneity exponent $(h=0.5)$ is characteristic of an effective one-dimensional topology. On the other hand, our data are not consistent with a percolation-network topology for the Vycor pore network [13]. To test this we have tested the percolation clusters of isotopic mixed alloys [24]. Previous mixed crystal data [24] showed that the heterogeneity factor increases below $12 \%$ napthalene $\left(\mathrm{C}_{10} \mathrm{H}_{8}\right.$ in $\mathrm{C}_{10} \mathrm{D}_{8}$ ) alloy concentrations (while above $12 \% h=0$ ). At low concentrations we now observe initial rate ratios higher than unity but below three, while at higher concentrations (39\%) we obtain a ratio of unity [36]. This is consistent with our simulations [36] (see also fig. 4).

The local density (early decay time) kinetic effect is thus a new criterion for the characterization of sample heterogeneity (we include low dimensionality as a special case of heterogeneity). We further note that the exciton transport in these samples is incoherent (hopping) [11].
A coherent transport would give different results. Obviously there is no mixing (stirring) mechanism available for our exciton ensembles. Homogeneous chemical kinetics implies efficient stirring (convection) and thus no local density effects. However, it is interesting to see that the diffusion-caused "self-stirring" [37] is sufficient to effectively quench the local density effect in the "homogeneous" lattices ( simple cubic - fig. 4, as well as square lattice and others [36]). Thus, exciton hopping in perfect (non-alloy) crystals is not expected to show the local density effect. Indeed, it is not observed.

As mentioned above, this new effect may also enable one to calibrate the absolute number of random hopping events within a given time period. The simulations indicate that the delayed fluorescence ratio (fig. 4) reaches unity after roughly $10^{2}$ "steps". Experimentally, this ratio of unity is achieved after about $0.1 \mathrm{~s}$. This again gives an average hopping time of about $1 \mathrm{~ms}$ (assuming effective one-dimensional topology).

We expect similar local density effects to appear in many analogous solid-state and "physical" reactions: Electron-hole recombination, defect annihilation or aggregation, and soliton-antisoliton annihilation. The same should be true for diffusion-limited solid-state and surface chemical reactions.

\section{Summary}

1) We have produced cylindrical molecular crystal wires down to a radius of $5 \mathrm{~nm}$ : The recombination process in- 
volves free and bound excitons ("heterofusion"). The triplet exciton kinetics fits a multiple-hopping model. The overall migration range is about $25 \mathrm{~nm}$ at $4 \mathrm{~K}$ and $40 \mathrm{~nm}$ at $77 \mathrm{~K}$. The long-time exciton transport is strictly onedimensional in the ultra-thin wires. The fractal-like kinetics model works well in a low-dimensional non-fractal system. 2) The porous-glass (Vycor) channels are described well by a non-fractal, quasi-one-dimensional topology. The naphthalene plugs in the Vycor pores are shown to be worm-like. These naphthalene-embedded Vycor pores also show a triplet exciton migration length of about 25 to $40 \mathrm{~nm}$. The Vycor sample exciton hopping time (per molecule), along the pore, is on the order of 1 ms. 3) The exciton annihilation method appears to be a reliable tool for probing spectral dimensions and low-dimensional topologies. A new method, based on initial local density effects is described. The initial density method is very sensitive to topology and hopping time.

\section{References}

[1] K. Lakatos-Lindenberg and K. E. Shuler, J. Math. Phys. 12 (1971) 633 .

[2] S. Alexander. J. Bernasconi. W.R. Schneider and R. Orbach, Rev. Mod. Phys. 53 (1981) 175.

[3] R. Zwanzig. J. Stat. Phys. 28 (1982) 127.

[4] V.M. Agranovich and M.D. Galanin, Electronic Excitation Energy Transfer in Condensed Matter (North-Holland. Amsterdam, 1982).

[5] A. Blumen, J. Klafter and G. Zumofen, in: Optical Spectroscopy of Glasses. ed. I. Zschokke (Reidel, Dordrecht. 1986) p. 199

[6] M. Buttiker, Y. Imry, R. Landauer and S. Pinhas, Phys. Rev. B31 (1985) 6207.

[7] A. Benoit, C.P. Umbach, R.B. Laibowitz and R.A. Webb, Phys. Rev. Lett. 58 (1987) 2343.

[8] D. Lenstra and W. van Haeringen. Phys. Rev. Lett. 57 (1986) 1623

[9] D.J. Thouless, Phys. Rev. Lett. 39 (1977) 1167.

[10] J. Hoshen and R. Kopelman, J. Chem. Phys. 61 (1974) 330.

[11] R. Kopelman, in: Modern Problems in Solid State Physics, Vol. 4. eds. V.M. Agranovich and R.M. Hochstrasser ( NorthHolland, Amsterdam, 1983) p. 139.

[12] D.W. Schaefer, B.C. Bunker and J.P. Wilcoxon. Phys. Rev. Lett. 58 (1987) 284
[13] U. Even, K. Rademann. J. Jortner, N. Manor and R. Reisfeld, Phys. Rev. Lett. 58 (1987) 285; ibid. 42 (1984) 2164

[14] W.D. Dozier, J.M. Drake and J. Klafter. Phys. Rev. Lett. 56 (1986) 197.

[15] R. Kopelman, S. Parus and J. Prasad, Phys. Rev. Lett. 56 (1986) 1742.

[16] C.L. Yang. P. Evesque and M.A. El-Sayed, J. Phys. Chem 89 (1985) 3442.

[17] R.M. Hochstrasser and Whiteman. J. Chem. Phys. 56 (1972) 5945.

[18] M.D Fayer, in: Modern Problems in Solid State Physics. Vol. 4 eds. V.M. Agranovich and R.M. Hochstrasser (NorthHolland. Amsterdam. 1983) p. 158.

[19] M.J. Burns. W.K. Liu and A.H. Zewail, ibid. p. 301.

[20] S.D.D.V. Rughooputh, D. Bloor. D. Phillip and B. Movaghar, Phys. Rev. B35 (1987) 8103. W.J. Rodriguez. R.A. Auerbach and G.L. McPherson. J Chem. Phys. 85 (1986) 6442

[21] R. Kopelman, J. Stat. Phys. 42 (1986) 185.

[22] S. Alexander and R. Orbach. J. de Phys. Lett. 43 (1982) L625.

[23] R. Rammal and G. Toulouse. J. de Phys. Lett. 44 (1983) L13.

[24] P.W. Klymko and R. Kopelman. J. Phys. Chem. 87 (1983) 4565 .

[25] C. Kittel. Introduction to Solid State Physics. 3rd, ed. (Wiley. New York. 1967).

[26] A. Suna, Phys. Rev. B14 (1970) 1716.

[27] G.R. Freeman. ed. Kinetics of Nonhomogeneous Processes (Wiley, New York (1987).

[28] T. Van Kampen, Stochastic Processes in Physics and Chemistry (North-Holland, Amsterdam, 1981).

[29] V. Kuzovkov and E. Kotomin. Chem. Phys. Lett. 117 (1985) 113

[30] R. Kopelman and S.J. Parus, in: Fractal Aspects of Materials, II. eds. D.W. Schaefer, R.B. Laibowitz, B.B. Mandelbrot and S.H. Liu. Materials Research Society. Boston (1986) p. 50.

[31] Nuclepore Corp. Pleasanton. CA

[32] J. Prasad and R. Kopelman, J. Lumin. (in press).

[33] L.W. Anacker and R. Kopelman, J. Chem. Phys. 81 (1984) 6402.

[34] L.W. Anacker. R.P. Parson and R. Kopelman. J. Phys Chem. 89 (1985) 4758

[35] L. Li and R. Kopelman, J. Lumin. (in press).

[36] S.J. Parus and R. Kopelman (unpublished).

[37] P. Argyrakis and R. Kopelman. J. Chem. Phys. 91 (1987) 2699. 